\title{
Maturating Articular Cartilage Can Induce Ectopic Joint-Like Structures in Neonatal Mice
}

\author{
Shinichirou Miura ${ }^{1,2} \cdot$ Rio Tsutsumi $^{3,4} \cdot$ Kiyokazu Agata $^{3,5} \cdot$ Tetsuya $^{\text {Endo }}{ }^{1}$ (D) \\ Received: 25 June 2020 / Revised: 14 September 2020 / Accepted: 24 September 2020/Published online: 6 October 2020 \\ (C) The Author(s) 2020
}

\begin{abstract}
Osteoarthritis is a huge health burden to our society. Seeking for potential ways to induce regeneration of articular cartilage (AC) that is intrinsically limited, we focused on the interaction between two opposing joints. To evaluate the role of the interaction of opposing regions of $\mathrm{AC}$ for joint maturation, we amputated digits at the distal interphalangeal level without injuring the articular surface of the intermediate phalanx (P2) and observed that the zonal organization of AC was defective. We then removed the P2 bone without injuring the articular surface of the proximal phalanx (P1), and the remaining part of the digit was amputated near the distal interphalangeal level. The distribution pattern of type II collagen and proteoglycan 4 (PRG4) suggested that maturation of AC in P1 was delayed. These two experiments suggested that an interaction between the opposing AC in a joint is necessary for maturation of the zonal organization of $\mathrm{AC}$ in neonatal digits. To test if an interaction of the joints is sufficient to induce articular cartilage, a proximal fragment of P2 was resected, inverted, and put back into the original location. Newly formed cartilage was induced at the interface region between the $\mathrm{AC}$ of the inverted graft and the cut edge of the distal part of P2. Type II collagen and PRG4 were expressed in the ectopic cartilage in a similar manner to normal AC, indicating that neonatal AC can induce ectopic joint-like structures in mice comparable with what has been reported in newts and frogs. These results suggest that the neonatal joint could be a source of inductive signals for regeneration of AC.
\end{abstract}

\section{Lay Summary}

In this study, we experimentally show that neonatal mice appear to have the capacity to regenerate articular cartilage (AC) in digits. It is already known that mice can regenerate a digit tip after amputation, but do not regenerate in response to amputations at more proximal levels. Therefore, it has been thought that mammalian joint structures are non-regenerative. However, we found that normal digit $\mathrm{AC}$ can induce $\mathrm{AC}$-like structures in a non-joint region when it is placed next to the cut edge of a bone, suggesting that the normal $\mathrm{AC}$ has regenerative capacity in certain situations in neonatal mice.

\section{Future Works}

Joint disorders are a huge health problem of our society. The results of this study suggest that neonatal AC could be a potential source of inductive signals for regeneration of AC. The discovery of these inductive signals will aid in developing regenerative therapies of a joint in human.

Keywords Joint $\cdot$ Articular cartilage $\cdot$ Digit $\cdot$ Limb $\cdot$ Regeneration $\cdot$ Repair

Electronic supplementary material The online version of this article (https://doi.org/10.1007/s40883-020-00176-w) contains supplementary material, which is available to authorized users.

Tetsuya Endo

tendo@dpc.agu.ac.jp

1 Division of Liberal Arts and Sciences, Aichi Gakuin University, Araike 12, Iwasaki-cho, Nissin, Aichi 470-0195, Japan

2 RENO Medical Healthcare Communications, Chuo-ku, Tokyo 104-0061, Japan
3 Department of Biophysics, Graduate School of Science, Kyoto University, Kyoto, Japan

4 Institute for the Advanced Study of Human Biology (WPI-ASHBi), Kyoto University, Kyoto 606-8501, Japan

5 Laboratory of Regeneration Biology, National Institute for Basic Biology, Okazaki, Japan 


\section{Introduction}

Synovial joints are a fundamental organ in skeletal function, which consists of opposing articular cartilage, tendons, ligaments, and synovium. Discovering how to regenerate joint structures is strongly desired because of its functional importance and the reality that articular cartilage (AC) deteriorates over time causing osteoarthritis. Currently, it is unclear why $\mathrm{AC}$ fails to be regenerated [1-3].

In urodele amphibians, it is known that functional joints are perfectly regenerated during regeneration of an amputated limb [4-7] and formation of accessory limbs [8], and also that partially removed AC can be repaired intrinsically [9]. In mammals, while fetal AC can be repaired from partial injury [10-12], adult AC has little regenerative ability, resulting in fibrocartilage formation in response to injury [13]. Recently, however, Yu et al. [14] have successfully stimulated joint regeneration in neonatal mouse digits by application of bone morphogenetic protein 9 (BMP9). These regenerated joints contain well-formed AC and tendon connections to the cartilage. This phenomenon probably reflects a function of BMP9 that is expressed in the joint interzone during limb development and evokes the developmental mechanisms. In addition, there are some other studies implying that there is a hidden potency to regenerate joint structures in adult mice [15-17].

The mouse digit is an important model to study epimorphic regeneration in mammals [18]. Epimorphic regeneration is defined as a response that involves the formation of a blastema [5]. In mice, a blastema can be formed only when a digit is amputated at the distal level of the distal phalanx (P3), whereas more proximal levels of a digit or limb are regenerationincompetent (for digit anatomy, see Fig. 1a) [16, 19-25]. Digit amputation at the proximal P3 level or the middle of the intermediate phalanx $(\mathrm{P} 2)$ results in a healing response without blastema formation and regeneration. Blastema formation, however, can be induced by delivery of BMPs to the amputation plane at these more proximal levels, resulting in the induction of bone regeneration $[22,26]$. At a mid P2 amputation, a BMP-induced blastema regenerates only the lost part of $\mathrm{P} 2$, but no more distal structures including the distal interphalangeal (DIP) joint. This is similar to what is seen in spike formation in an amputated limb of the African clawed frog, Xenopus laevis, which is a model system of incomplete limb regeneration [27-29]. In Xenopus, when a forelimb is amputated at the middle of the forearm, a blastema is formed on the amputation plane that develops into a cartilaginous rod, called a spike, which does not contain joint structures [28, 30, 31].

Recently, it was shown that Xenopus has the ability to regenerate a functional elbow joint in response to a hemiarticular amputation at the elbow level with the distal $\mathrm{AC}$ of a humerus remaining intact [32]. A joint is regenerated between the blastema-derived cells and the remaining $\mathrm{AC}$ of the stump. In joint development, to develop the mature interlocking structure, reciprocal interactions between opposing skeletal elements are needed [33]. It is thus suggested that there is a comparable morphogenetic interaction between a blastema and a bone of the stump in the Xenopus experiment, which is called "reintegration" $[7,32]$ In the mouse digit model, however, this has never been observed, because a blastema is not formed at any joint level in response to amputation, and therefore, the regenerative capacity of the stump tissues has yet to be evaluated.

In this study, we examined whether there are interactions between the opposing AC in a joint in neonatal mice. Mature $\mathrm{AC}$ has well-defined horizontal cell layers that are organized during postnatal development [34-36]. Though digital AC is thinner and has less obvious zonal organization than that of large joints such as a knee, at least three layers are distinguishable and can be visualized by the distribution of type II collagen and proteoglycan 4 (PRG4) proteins. By surgically manipulating the joint tissues and examining the distribution of these proteins, we have discovered that $\mathrm{AC}$ in neonatal mouse digits has the ability to interact with the opposing $\mathrm{AC}$ in a joint to maturate its zonal organization, and also that neonatal AC can induce an ectopic AC-like structure (a joint) at the cut edge of a broken bone.

\section{Materials and Methods}

\section{Ethics Statement}

All animal experiments were performed in accordance with protocols approved by the Institutional Animal Care and Use Committee of Aichi Gakuin University, Division of Liberal Arts and Sciences.

\section{Animals}

ddY mice were purchased from Chubu Kagaku Shizai Co., Ltd. (Nagoya, Japan). Parental mice were bred and maintained under standard conditions in the laboratory, and neonatal mice were used in experiments.

\section{Surgical Procedures}

Newborn mice were anesthetized by placing on ice for $10 \mathrm{~min}$ for the following surgical procedures. Hind limb digit 3 in postnatal day 2 (PN2) and PN1 neonates was used for hemiarticular amputation and other experiments, respectively. For hemiarticular amputation, skin at the distal interphalangeal (DIP) level was incised circumferentially. The P3 skeletal element was removed by cutting off the connection between P3 and P2 (Fig. 1b). For hemiarticular resection at the proximal interphalangeal (PIP) level, ventral skin at P2 and P1 levels was incised proximodistally; and inner tissues were 
Fig. 1 Illustration of the experimental surgeries in this study. (a) A normal hind limb digit. (b) Hemiarticular amputation at the DIP level. (c) Hemiarticular resection at the PIP level. (d) Proximal P2 inverted experiment and its sham operation
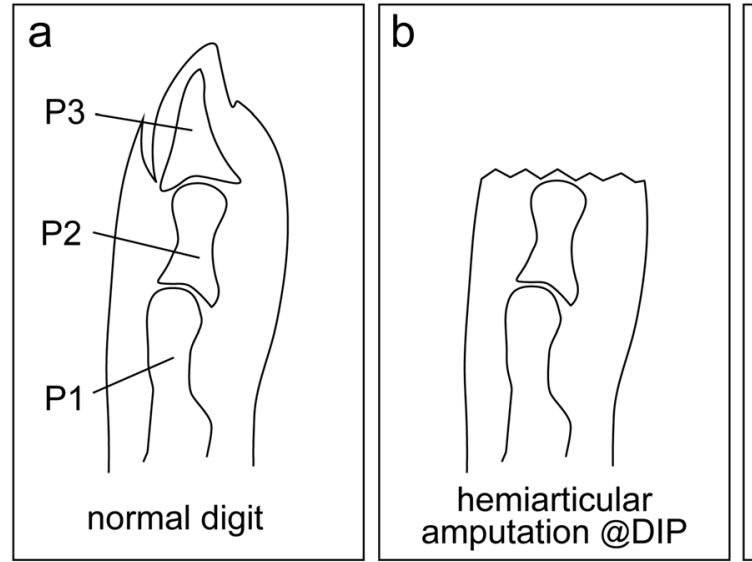

C

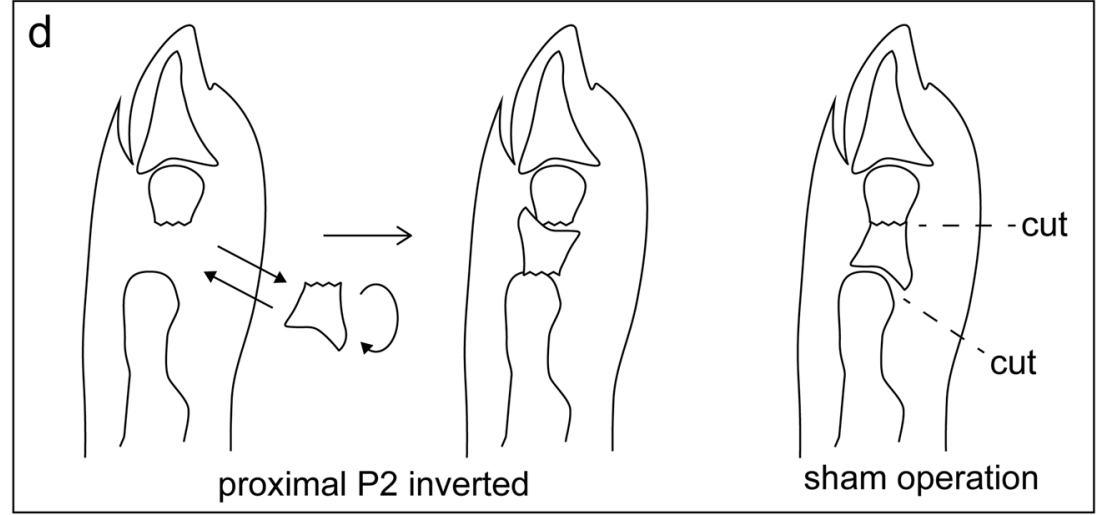

exposed. The digital flexor tendon was severed at the PIP joint. The tip of the surgical knife was inserted into the cavity between $\mathrm{P} 2$ and the proximal phalanx (P1). Careful attention was paid not to injure the articular surface of P1. The P2 skeletal element was also severed in the middle, and the proximal half of $\mathrm{P} 2$ was removed by cutting off the terminal extensor tendon. Finally, the digit was amputated near the DIP level; and the distal fragment of P2 bone was removed (Fig. 1c). For the proximal $\mathrm{P} 2$ inverted experiment, a proximal fragment of $\mathrm{P} 2$ was pulled out as described above, inverted proximodistally, and grafted back into the original position (Fig. 1d). As control experiments for hemiarticular resection and proximal P2 inverted experiment, sham operations were made in which the proximal half of P2 was severed but not inverted (Fig. 1d).

After each surgery, we immediately applied Terramycin ointment (Yoshindo, Toyama, Japan) and Tegaderm Film (3M Health Care, Neuss, Germany) to the skin incision for care.

\section{Histology}

Digit samples were fixed in $10 \%$ formalin-PBS overnight at room temperature (RT). Fixed samples were washed in PBS and decalcified in Morse's solution (10\% sodium citrate and $22.5 \%$ formic acid) for 1-2 days at RT, processed, embedded in paraplast (Sigma), and sectioned
(10 $\mu \mathrm{m})$. Tissue sections were deparaffinized, hydrated, and processed as follows. Mallory's trichrome staining was performed as described by Humason [37]. For hematoxylin, eosin and alcian blue staining, tissue sections were treated with $3 \%$ acetic acid, and stained with $1 \%$ alcian blue 8GX (Sigma). After alcian blue staining, slides were washed in $3 \%$ acetic acid and PBS and processed for Mayer's hematoxylin (Wako) and eosin Y (Wako) staining.

\section{Immunohistochemistry}

For immunohistochemistry, tissue sections were deparaffinized and hydrated in Tris-buffered saline (TBS). Sections were treated with $10 \mu \mathrm{g} / \mathrm{mL}$ proteinase $\mathrm{K}$ (Invitrogen) in TBS for $20 \mathrm{~min}$ at $37^{\circ} \mathrm{C}$. After treating with Blocking One Histo (Nacalai tesque, Kyoto, Japan) for $10 \mathrm{~min}$ at RT, sections were incubated with rabbit anti-collagen II polyclonal antibody (Bioworld Technology, \#BS1071, diluted 1:400) or rabbit antilubricin (PRG4) polyclonal antibody (Abcam, \#ab28484, diluted 1:250) overnight at $4{ }^{\circ} \mathrm{C}$. After primary antibody incubation, sections were incubated with anti-rabbit IgG-alkaline phosphatase (Sigma, \#A3937, diluted 1:100) for $60 \mathrm{~min}$ at RT. Signal was detected by NBT/BCIP (Sigma). 


\section{Results}

\section{Digit Amputation at the Interphalangeal Level Disrupts the Maturation of Articular Cartilage}

Maturation of AC occurs postnatally in mouse digits. The ossified area expands from the center of the P2 bone toward both the distal and proximal ends; however, the distal epiphysis is still cartilaginous at PN6 (Fig. 2a). AC is distinguishable according to its staining by aniline blue. It becomes morphologically more obvious by PN16, because it contains three layers in AC: the outer layer where round-shaped cells are orderly aligned, the intermediate layer that consists of flatshaped cells, and the inner layer that is ossified (Fig. 2b). The inner layer is more ossified by PN30 (Fig. 2c).

In order to test if the interaction of two opposing joints is necessary in the maturation process of $\mathrm{AC}$, we amputated digits at the DIP level, leaving the articular surface of P2 intact (Fig. 1b). By 4 days post-amputation (dpa), no indication of blastema formation is observed around the amputation plane that is covered by the epidermis and thick connective tissue layer (Fig. 2d). However, AC is less obvious than that of the normal digit. At $14 \mathrm{dpa}$, the articular chondrocytes are not histologically distinguishable from other epiphyseal chondrocytes, and ossification is delayed in the epiphysis (Fig. 2e). The remaining deep digital flexor tendon and terminal extensor tendon regenerate underneath the wound epidermis and connect with each other. Some collagen fibers from the tendons connect to the articular surface of the distal P2 bone or the distal interphalangeal sesamoid bone. At $28 \mathrm{dpa}$, although the marginal area of the epiphysis remains cartilaginous, chondrocytes are irregularly distributed, compared with ones in normal AC (Fig. 2f). Tendon connection to the articular surface is more drastic than 14-dpa-samples. These results were obtained in all digits analyzed $(n=3$ for 4-dpa and 14dpa digits, and $n=2$ for 28-dpa digits).

These results suggest that interaction of the opposing joints is necessary for proper maturation of AC. However, it was not clear whether the failure in AC maturation is caused by the lack of the opposing AC per se, disruption of the joint tissues surrounding $\mathrm{AC}$ such as the synovial membrane and fibrous capsule of a joint capsule that is filled with the synovial fluid, or exposure of $\mathrm{AC}$ to the external environment due to the amputation.

\section{Interaction Between the Opposing Joint Elements Is Necessary for the Maturation of Articular Cartilage}

For further investigation, we modified the experimental surgery and performed hemiarticular resection at the PIP level. In this experiment, the $\mathrm{P} 2$ skeletal element was resected leaving the distal epiphysis of P1 intact; and then the digit was amputated near the DIP level (Fig. 1c). Therefore, the distal diaphysis of P1 is surrounded by skin and other soft tissues of P2 level; and it is expected that the exposure of AC to the external environment can be minimized and that it is easy to perform the sham operation as control. We also observed molecular marker distribution of AC to compare detailed histology with those in normal digits.
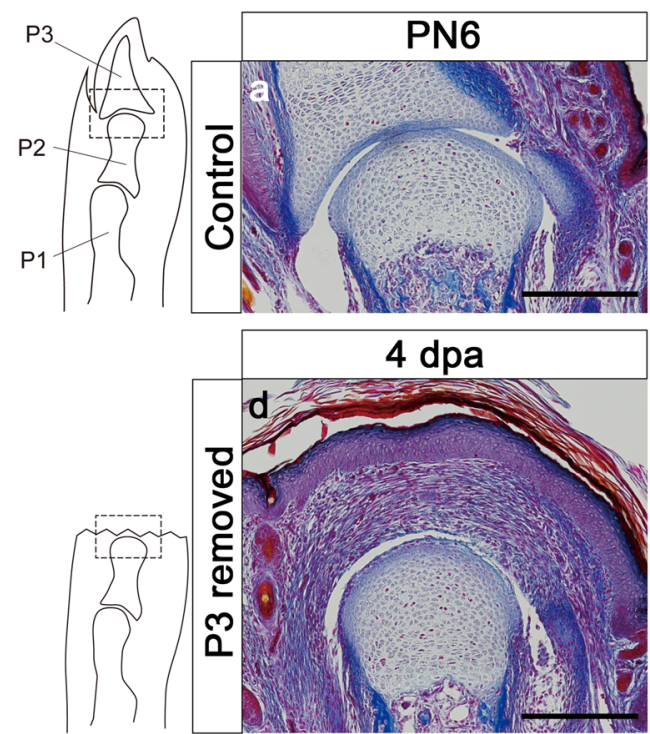

Fig. 2 Hemiarticular amputation at distal interphalangeal level. Tissue sections were stained by Mallory's trichrome staining. (a-c) DIP joints of normal digits at PN6 (a), $16(\mathbf{b})$, and $30(\mathbf{c})$. (d-f) DIP joints of hemiarticular amputated digits 4 dpa (d), 14 dpa (e), and 28 dpa (f).
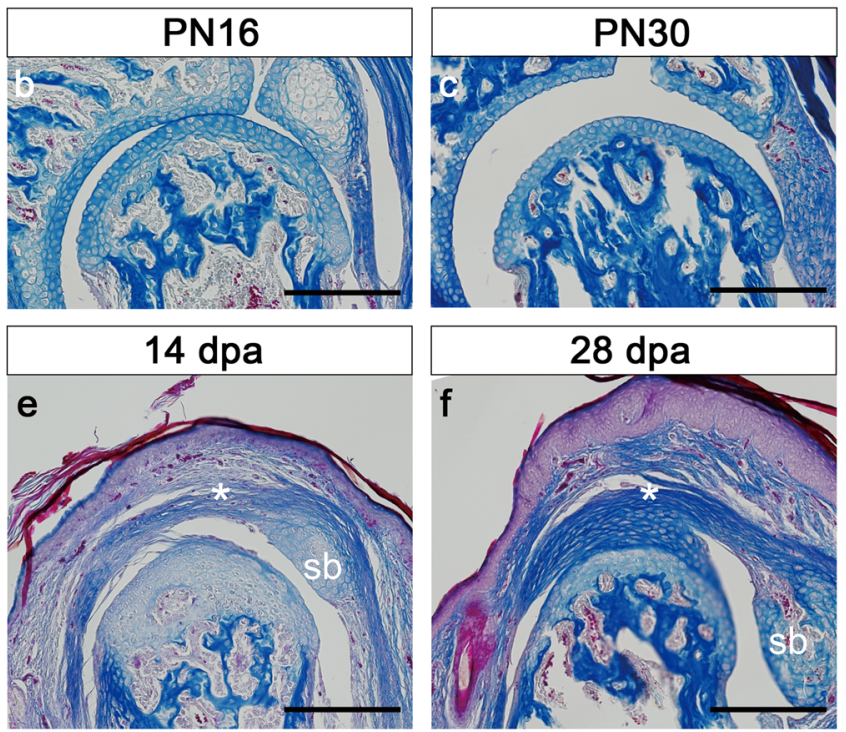

The dashed squares in the illustrations indicate photographed areas. sb: distal interphalangeal sesamoid bone, asterisk: the connected site between the remaining deep digital flexor tendon and terminal extensor tendon. Scale bars $=200 \mu \mathrm{m}$ 
In normal digits, immunohistochemistry of type II collagen and PRG4 revealed that three layers of AC are gradually organized by about 3 weeks of age. At PN8, AC is distinguishable from the epiphyseal hypertrophic cartilage in cell shape (Fig. 3a) and expresses type II collagen (Fig. 3b). The signal is detected in both the cartilage lacunae and matrix region. PRG4 is localized in the outer layer of AC and partially in the articular surface (Fig. 3c). The flatted-shaped signals are also observed in the bone marrow. Although zonal organization of $\mathrm{P} 1$ is not as obvious as that of the distal epiphysis of $\mathrm{P} 2$ (Fig. 3d and g, compared with Fig. 2b and c), the distribution of type II collagen is eventually localized to the inner layer of $\mathrm{AC}$ and also found in the central slip of the terminal extensor tendon and the volar plate (Fig. $3 \mathrm{e}$ and $\mathrm{h}$ ). The PRG4 signal is detected in the superficial single cell layer, the articular surface of the whole epiphysis, and the synovial membrane (Fig. $3 \mathrm{f}$ and $\mathrm{i}$ ). Therefore, it takes at least 3 weeks to develop mature $\mathrm{AC}$ of a PIP joint in mice after birth.

During the maturation of $\mathrm{AC}$, interactions between the opposing AC might play important roles because zonal organization of $\mathrm{AC}$ is disturbed in the P1 distal epiphysis of hemiarticularly resected PIP joints. As seen in hemiarticular DIP amputation, regenerated tendons from the dorsal and ventral sides fuse each other and some of them connect to the P1 distal epiphysis (Fig. 4a and d). Type II collagen is still distributed throughout AC in 14 days post-surgery (dps; Fig. 4b), although it starts shifting toward the intermediate layer in the normal joint (see Fig. 3e). The signal is also seen in the connecting tendons, indicating that the attachment site consists of fibrocartilages (Fig. 4b). PRG4 is localized in the superficial cell layer of $\mathrm{AC}$ and the articular surface as well as in the normal joint, although signal strength is weak (Fig. 4c). This distribution pattern of type II collagen and PRG4 continues by $21 \mathrm{dps}$ (Fig. 4e and f). On the other hand, the sham operation resulted in normal histology and distribution of type II collagen and PRG4 (Fig. 4g-i). These results were obtained in all digits analyzed ( $n=3$ for 14-dps and 21-dps digits, and $n=4$ for sham-operated digits).

In hemiarticular resection, the exposure of $\mathrm{AC}$ to the external environment is reduced, compared with DIP amputation. The result of the control surgery indicates that the disruption of the joint capsule does not affect the maturation of AC. Taken together, it is suggested that $\mathrm{AC}$ in the opposing joint is necessary for the maturation of AC.

\section{Maturating Articular Cartilage Can Induce Joint-Like Structures and Molecular Marker Expression}

To test if AC can interact with the opposing AC to maintain its maturation and also induce joint structures in a non-joint region, we performed the following experiment. The proximal half of the $\mathrm{P} 2$ bone was resected, inverted, and put back to the original position (Fig. 1d). After the surgery, the P2 proximal $\mathrm{AC}$ faces the cut edge of the distal half of $\mathrm{P} 2$, where cartilaginous callus is supposed to be formed as a fracture response;
Fig. 3 Postnatal maturation of articular cartilage in the proximal interphalangeal joint at PN8 (ac), $15(\mathbf{d}-\mathbf{f})$ and $22(\mathbf{g}-\mathbf{i})$. Hematoxylin, eosin, and alcian blue staining (a, d, g). Immunohistochemistry for type II collagen (b, e, h) and PRG4 (c, f, i). Scale bars $=100 \mu \mathrm{m}$
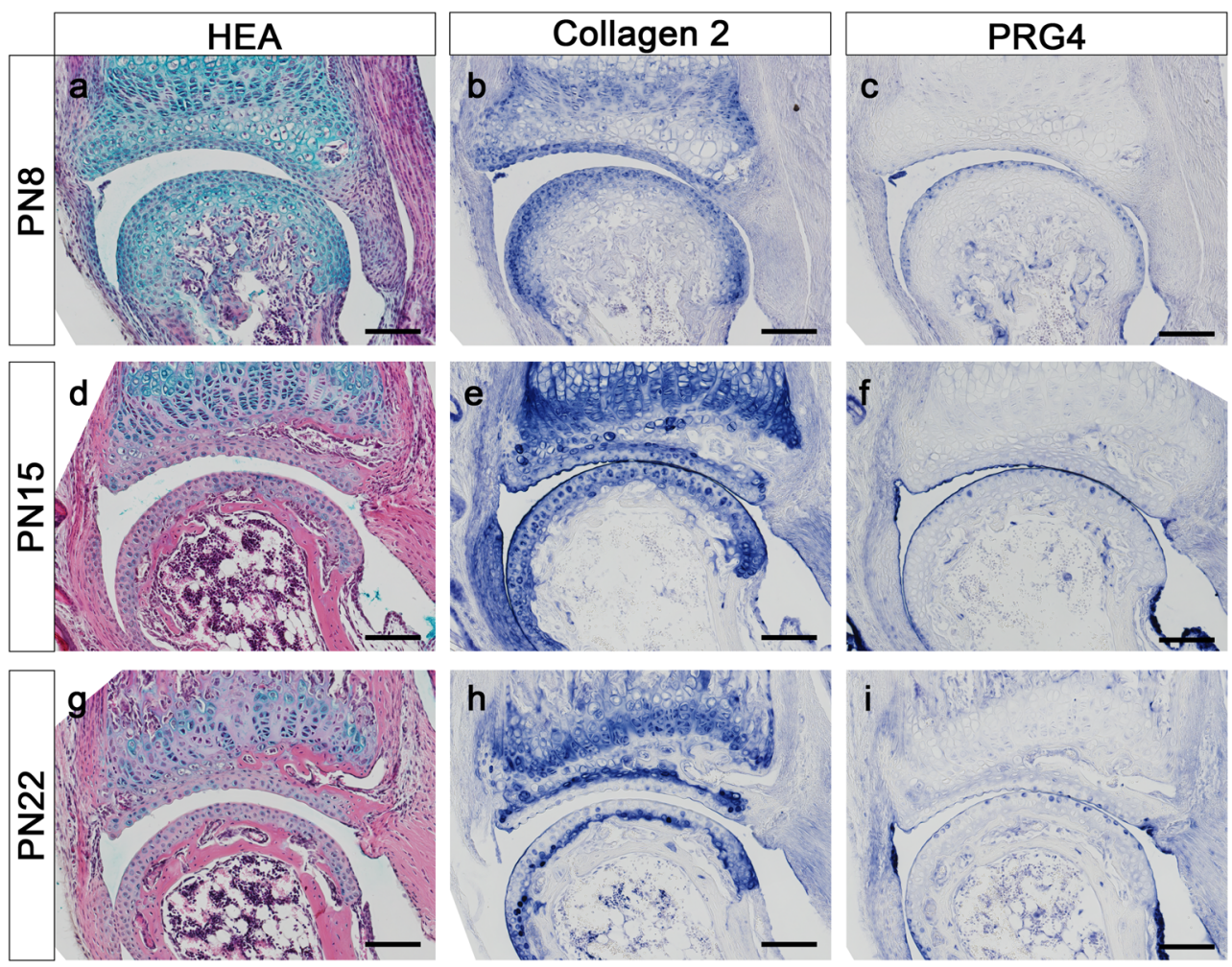


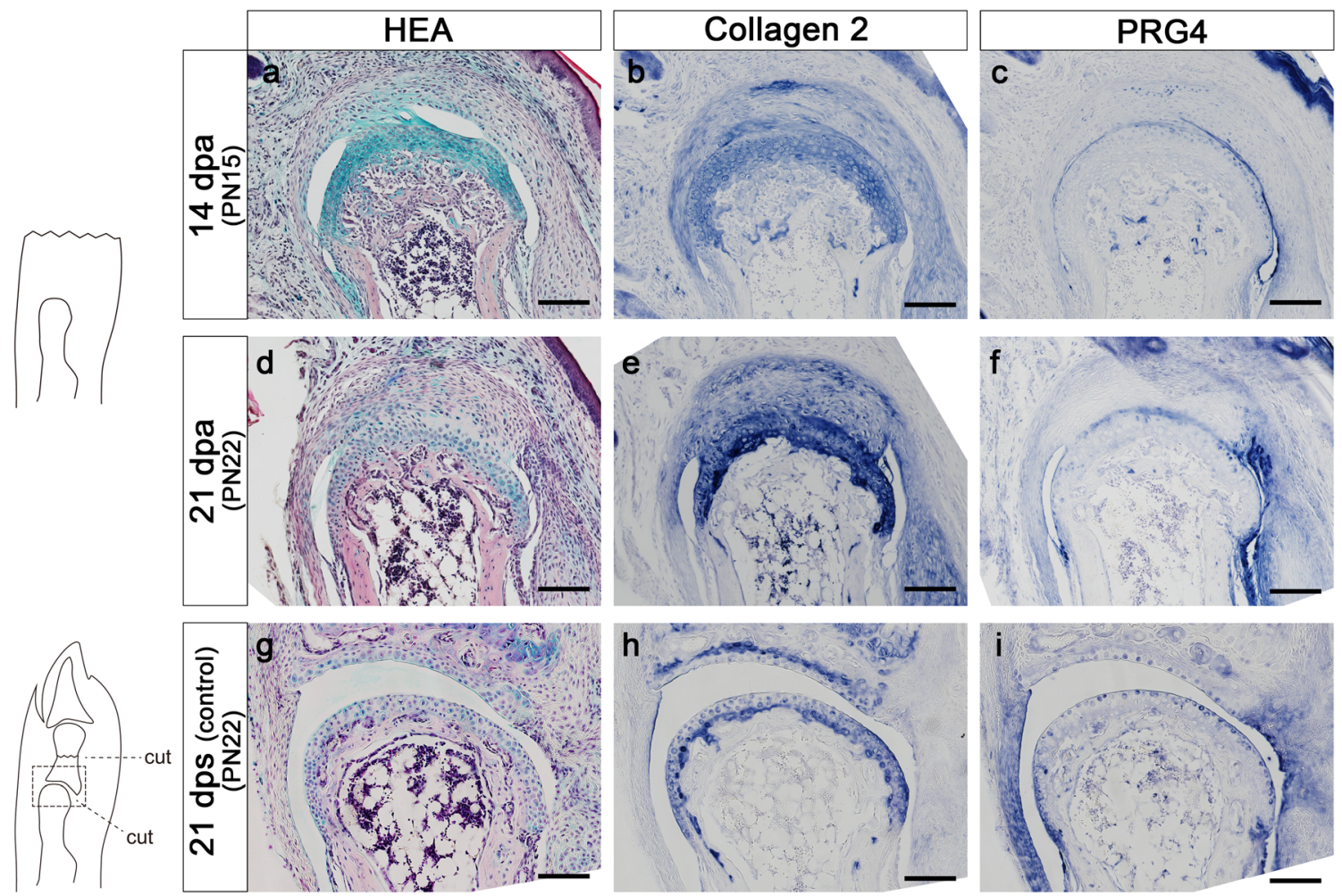

Fig. 4 Hemiarticular resection at the PIP level at $14 \mathrm{dps}(\mathbf{a}-\mathbf{c})$ and $21 \mathrm{dps}(\mathbf{d}-\mathbf{f})$. The control is shown in $\mathbf{g}-\mathbf{i}$. (a, d, g) Hematoxylin, eosin, and alcian blue staining. Immunohistochemistry for type II collagen $(\mathbf{b}, \mathbf{e}, \mathbf{h})$ and PRG4 $(\mathbf{c}, \mathbf{f}, \mathbf{i})$. Scale bars $=100 \mu \mathrm{m}$

and we observed the interface region (the dashed squared area in Fig. 5).

At $21 \mathrm{dps}$, fracture repair responses occur at the amputation site of $\mathrm{P} 2$ in the sham-operated digits, and the distal and proximal half of $\mathrm{P} 2$ fragments are bridged by the fibrocartilaginous tissue (Fig. 5a and c). Type II collagen-positive regions are scattered in the P2 bone, each of which is small (Fig. 5c). Although some PRG4positive cells are found around the plane of contact between the fragments, only a few PRG4-positive cells are found adjacent to type II collagen-positive regions, and the signal is weak (Fig. 5e). PRG4 signal is also seen in the peripheral layer of the bones, which is the synovial membrane of the DIP joint. In contrast, in the proximal P2-inverted digits, a synovial-like cavity is present between the fragments (Fig. 5b). An ectopic type II collagen-positive region appears at the cut edge of the distal P2 fragment along the boundary with the proximal $\mathrm{AC}$ of the inverted fragment (arrowhead region in Fig. 5d). Several PRG4-positive cells are found associated with the ectopic type II collagen region, and the signal is sharper compared with those in the sham experiment (Fig. 5f). These results were obtained in 3 digits out of 4 for the sham operation and in 2 digits out of 3 for the inverted experiment. Robust PRG4 staining is also seen in the area that lines the cavity between the fragments and in the connective tissues in the cavity. In the negative case of the inverted experiment, the inverted graft was dislocated from the appropriate position; and thick connective tissue formed between the inverted graft and the distal P2 fragment.

The operated digits at 56 dps display even more obvious ectopic joint-like structures. The ectopically formed cartilages at the cut edge of the distal P2 remain on the opposite side of the cavity from $\mathrm{AC}$ of the inverted fragment (Fig. $5 \mathrm{~g}$ and h). The distribution of type II collagen demonstrates that the ectopic cartilages consist of zonal organization like normal AC. In the inner layer, type II collagen is distributed in both the cartilage lacunae and matrix region, although it is seen only in the lacunae in the outer layer (Fig. 5i). PRG4-positive cells are localized in the superficial layer of the ectopic cartilage in the vicinity of the inverted graft (Fig. 5j; see also Supplemental Fig. 1). These characteristics of the experimental result are comparable with those seen in the normal joint. These results were obtained in all digits analyzed $(n=4)$. The robust PRG4 expression is observed in the layer that lines the cavity and the connective tissues.

The same results were obtained in the interface region between the cut edge of the proximal P2 fragment and $\mathrm{AC}$ in the distal epiphysis of $\mathrm{P} 1$ (the asterisked area in Fig. $5 \mathrm{~g}$ ). However, we think that analyses at this region should be made more carefully in further studies because we cannot eliminate the possibility that part of the $\mathrm{AC}$ in the proximal epiphysis of P2 remains at the PIP level after the surgery and participates in 

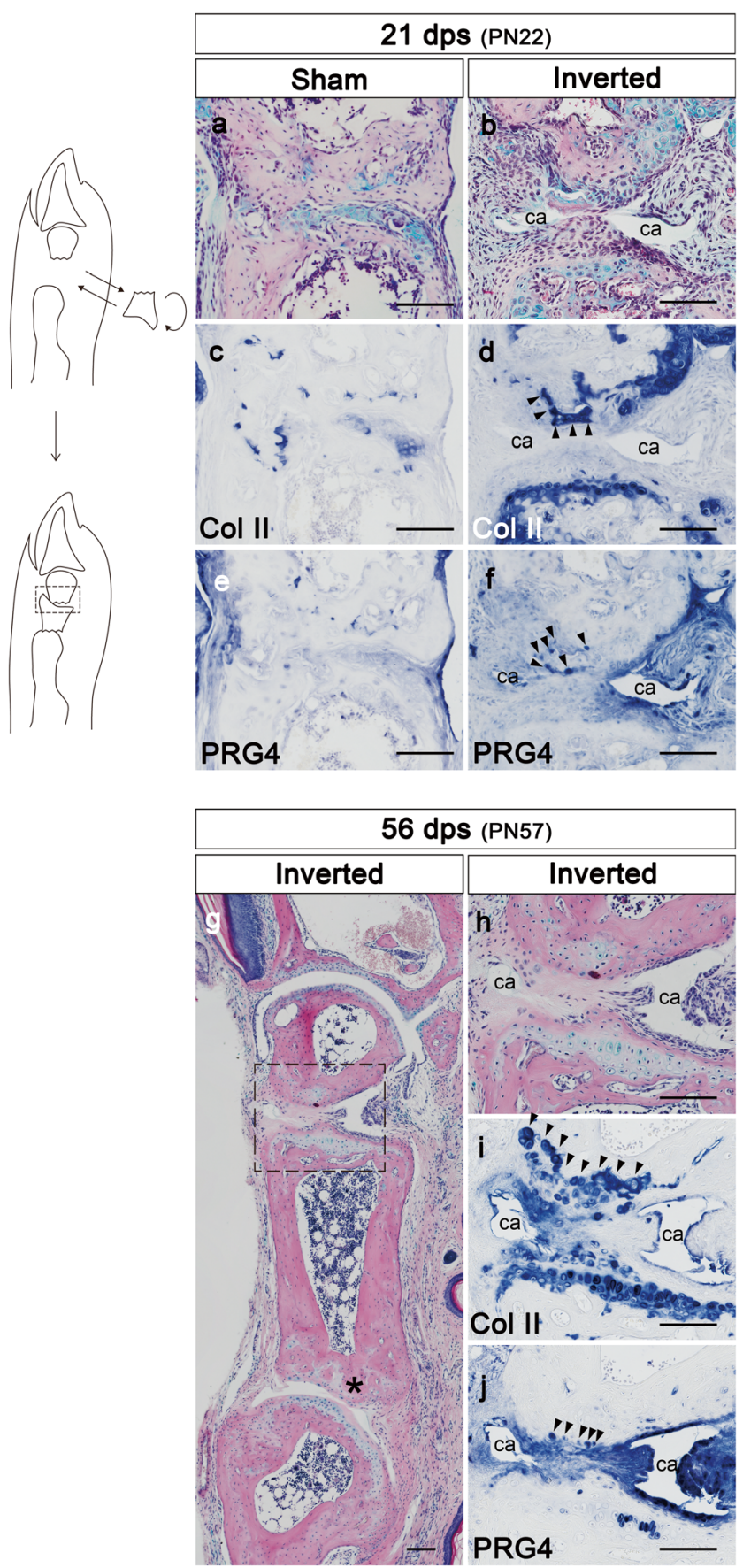

Fig. 5 Proximal P2-inverted experiment at $21 \mathrm{dps}(\mathbf{a}-\mathbf{f})$ and $56 \mathrm{dps}(\mathbf{g}-\mathbf{j})$. $(\mathbf{a}, \mathbf{c}, \mathbf{e})$ Fracture repair in a sham-operated P2 digit. $(\mathbf{b}, \mathbf{d}, \mathbf{f}, \mathbf{h}-\mathbf{j})$ The adjoining area of the cut edge of the distal P2 fragment and articular cartilage of the inverted proximal P2 fragment (dashed squares in the illustration and $\mathbf{g})$. (a, $\mathbf{b}, \mathbf{g}, \mathbf{h})$ Hematoxylin, eosin, and alcian blue staining. Immunohistochemistry for type II collagen $(\mathbf{c}, \mathbf{d}, \mathbf{i})$ and PRG4 $(\mathbf{e}, \mathbf{f}, \mathbf{j})$. Arrowheads in $\mathbf{f}$ and $\mathbf{j}$ indicate PRG4-positive cells in ectopically induced articular cartilage-like structure. The asterisked area in $\mathbf{g}$ indicates the interface region between the cut edge of the proximal P2 fragment and $\mathrm{AC}$ in the distal epiphysis of P1. ca: the cavity between the fragments. Scale bars $=100 \mu \mathrm{m}$

the formation of the ectopic cartilage. Therefore, in the current study, we focused only on the interface region between AC in the proximal epiphysis of $\mathrm{P} 2$ and the cut edge of the distal P2 fragment as shown in Fig. $5 \mathrm{~h}-\mathrm{j}$.

\section{Discussion}

There are several reasons that mice are thought to be unable to regenerate joint structures. The first is that mouse digit regeneration is position-dependent and only the tip of the distalmost bone can regenerate. A blastema cannot be formed at more proximal level, where joints are seen $[24,26]$. Since the blastema is a cellular source of epimorphic regeneration $[38,39]$, the inability to form a blastema naturally means no regeneration of any distal structures to the amputation plane. Secondly, even though blastema formation is inducible by the treatment of the amputation wound with BMP2, the blastema fails to regenerate a joint and distal skeletal element $[22,40]$. BMP2-enhanced regeneration is limited intrasegmentally and results in formation of a single bone segment. This is similar to spike formation that is an incomplete regenerative response to limb amputation in Xenopus laevis [28, 30, 31]. A spike cartilage is continuous with the remaining bone in the stump when a limb is amputated in the middle of the long bone, such as the radio-ulna. On the other hand, when a limb is amputated at the elbow level with distal $\mathrm{AC}$ of a humerus remaining intact, a joint is regenerated between the blastema-derived cartilage and remaining $\mathrm{AC}$ of the stump, suggesting that intact $\mathrm{AC}$ has the ability to interact with chondrogenic precursors to induce ectopic AC [7, 32]. In mice, however, whether the $\mathrm{AC}$ has the same ability has not been tested because a blastema is not formed after hemiarticular amputation at such as the DIP level.

In this study, first, we showed that neonatal $\mathrm{AC}$ has the ability to interact with opposing $\mathrm{AC}$ of a joint in mouse digits, and that this ability might be involved in formation of an ectopic AC-like structure (Fig. 6). The apposition of articular cartilages across the synovial cavity is necessary for postnatal maturation of joints. Without articular cartilage on one side, maturation of $\mathrm{AC}$ on the other side seems to be delayed, suggesting that they interact with each other to develop mature structures (Fig. 6a). In addition, when intact AC is placed by the cut edge of an amputated bone, type II collagen and PRG4 proteins are observed in the cartilaginous callus formed at the bone edge in an $\mathrm{AC}$-specific manner (Fig. 6b). Callus formation is the default fracture healing response [41, 42], and the fracture callus in the control experiment does not show AClike distribution pattern of type II collagen and PRG4. AC-like distribution is only seen in the vicinity of the intact $\mathrm{AC}$ of the inverted graft. Thus, local interaction appears to be involved in this induction (Fig. 6b).

We are currently assuming that the mechanism of ectopic $\mathrm{AC}$ induction is related to that of postnatal maturation of normal AC. During postnatal maturation, AC acts on 
Fig. 6 Schematic illustration of the experimental results. (a) Interaction between opposing articular cartilage is necessary for their postnatal maturation. Without opposing articular cartilage, postnatal maturation delays. (b) Normal articular cartilage induces ectopic articular cartilage-like structures in the fracture callus. The area of light blue indicates type II collagenpositive region in articular cartilage. The red spots indicate PRG4-positive cells in articular cartilage a

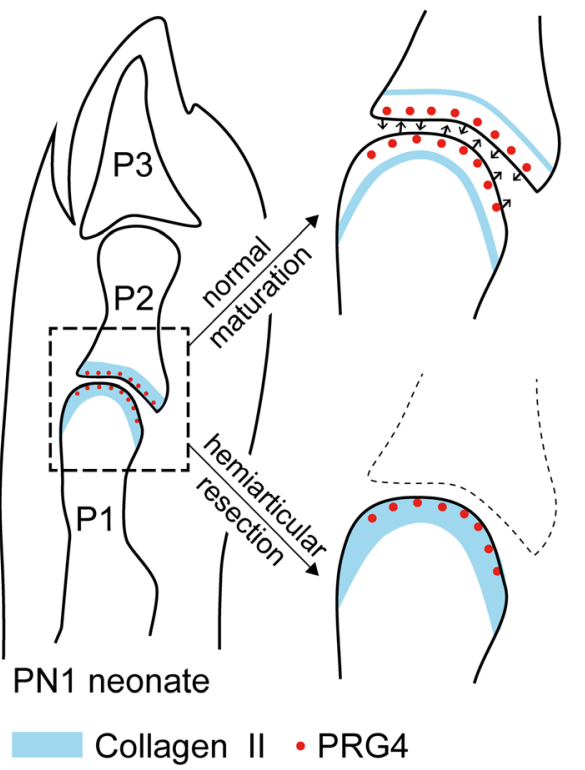

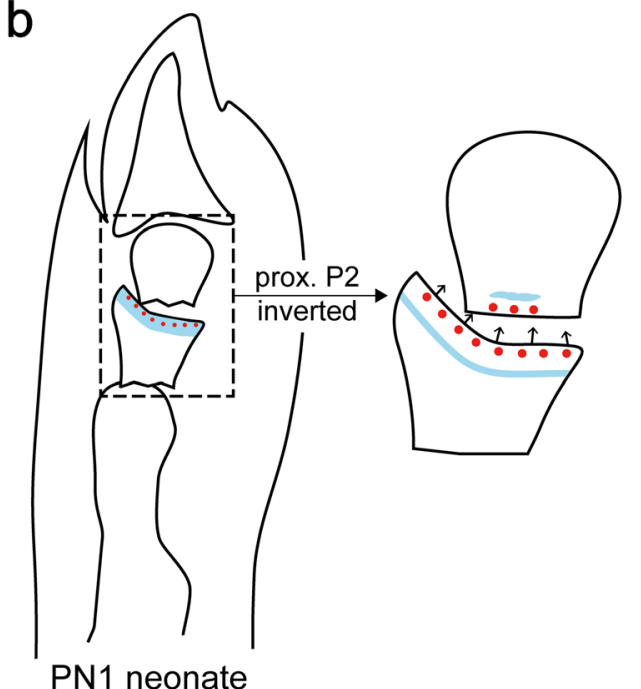

adjacent tissues across a joint cavity; therefore, presumably soluble molecules participate in these interactions. AC consists of chondrocytes surrounded by extracellular matrix that contains many sorts of growth factors, such as TGF- $\beta$ and BMPs [43]. TGF- $\beta$ and BMPs are suggested to be essential regulators of joint formation by many studies [44]. Joint-specific conditional knockout of BMP receptor 1a shows skeletal defects in some joints and failure to maintain AC in others postnatally [45]. Postnatal disruption of TGF- $\beta$ and BMP signaling results in a decrease of proteoglycan content in $\mathrm{AC}$ [46]. It will be important to examine the signaling of these molecules in our experimental system for further understanding the mechanism of ectopic joint induction. Although mechanical stimuli are also to be considered because movement-related mechanical stimuli are known to be essential in synovial joint formation [47], we assume that the biochemical stimuli is more likely to play roles in our experiments, since there is no evidence that significant movement occurs at the surgery site.

The second concern is whether the interzone-mediated mechanism is involved in ectopic AC formation or not. The interzone is a signaling center of joint formation and provides cells to AC and other joint tissues [33, 36, 48, 49]. In the proximal P2-inverted experiment, some surrounding connective tissues migrate into the space between the distal P2 fragment and the inverted proximal P2 fragment, which might form an interzone-like signaling center to induce ectopic AC. In the Xenopus hemiarticular amputation study, gene expression of GDF5, which is normally expressed in the interzone of a developing joint, is weak at the connecting site between cartilages derived from the blastema and the stump, but rather strong in cartilaginous regions [32]; therefore, whether the interzone is regenerated or not is still unclear. We need to examine expression and function of the molecules that play important roles in the interzone-mediated mechanism during formation of ectopic AC in the future studies.

It is also noteworthy that not only AC but also other tissues show signs of joint regeneration. In the proximal P2 inverted experiment, robust PRG4 expression is observed in the layer that lines the cavity between the P2 bone fragments and in the connective tissues, which is suggestive of synovial membrane regeneration. Tendon connection to the articular surface that is seen in hemiarticular amputation and resection is also an intriguing phenomenon for studying regeneration of the enthesis, which is an essential connective tissue for transmission of force between bone and tendon/ligament. Understanding regenerative responses of multiple tissues and how they can be coordinated will be required for the achievement of regeneration of the joint as an organ.

In conclusion, it appears that neonatal mouse digits innately demonstrate joint regenerative response in certain situations. BMP9 treatment might enhance and organize this hidden potency, resulting in successful joint regeneration [14]. Exploring AC-derived signaling is required for further understanding the mechanism of joint regeneration.

Acknowledgments We thank David Gardiner and Susan Bryant for their critical reading of the manuscript. We also thank Ken Muneoka, Haruka Matsubara, Miyuki Suzuki, and Ken-ichi Suzuki for discussion.

Funding This work was supported by JSPS KAKENHI Grant Numbers 18K06270 to TE and 16H06376 to KA from the Ministry of Education, Science, Sports, and Culture of Japan. 


\section{Compliance with Ethical Standards}

Conflict of Interest The authors declare that they have no conflict of interest.

Open Access This article is licensed under a Creative Commons Attribution 4.0 International License, which permits use, sharing, adaptation, distribution and reproduction in any medium or format, as long as you give appropriate credit to the original author(s) and the source, provide a link to the Creative Commons licence, and indicate if changes were made. The images or other third party material in this article are included in the article's Creative Commons licence, unless indicated otherwise in a credit line to the material. If material is not included in the article's Creative Commons licence and your intended use is not permitted by statutory regulation or exceeds the permitted use, you will need to obtain permission directly from the copyright holder. To view a copy of this licence, visit http://creativecommons.org/licenses/by/4.0/.

\section{References}

1. Ambrosi TH, Longaker MT, Chan CKF. A revised perspective of skeletal stem cell biology. Front Cell Dev Biol. 2019;7:189.

2. Li MH, Xiao R, Li JB, Zhu Q. Regenerative approaches for cartilage repair in the treatment of osteoarthritis. Osteoarthr Cartil. 2017;25:1577-87.

3. van der Kraan PM. The interaction between joint inflammation and cartilage repair. Tissue Eng Regen Med. 2019;16:327-34.

4. Wallace H. Vertebrate limb regeneration. New York: John Wiley and Sons Ltd.; 1981.

5. Carlson BM. Principles of regenerative biology. New York: Academic Press; 2007.

6. Lee J, Gardiner DM. Regeneration of limb joints in the axolotl (Ambystoma mexicanum). PLoS One. 2012;7:e50615.

7. Tsutsumi R, Inoue T, Yamada S, Agata K. Reintegration of the regenerated and the remaining tissues during joint regeneration in the newt Cynops pyrrhogaster. Regeneration. 2015;2:26-36.

8. Endo T, Bryant SV, Gardiner DM. A stepwise model system for limb regeneration. Dev Biol. 2004;270:135-45.

9. Cosden RS, Lattermann C, Romine S, Gao J, Voss SR, MacLeod $\mathrm{JN}$. Intrinsic repair of full-thickness articular cartilage defects in the axolotl salamander. Osteoarthr Cartil. 2011;19:200-5.

10. Namba RS, Meuli M, Sullivan KM, Le AX, Adzick NS Spontaneous repair of superficial defects in articular cartilage in a fetal lamb model. J Bone Joint Surg Am. 1998;80:4-10.

11. Kumahashi N, Ochi M, Kataoka H, Uchio Y, Kakimaru H, Sugawara K, et al. Involvement of ATP, increase of intracellular calcium and the early expression of c-fos in the repair of rat fetal articular cartilage. Cell Tissue Res. 2004;317:117-28.

12. Ribitsch I, Mayer RL, Egerbacher M, Gabner S, Kanduła MM, Rosser J, et al. Fetal articular cartilage regeneration versus adult fibrocartilaginous repair: Secretome proteomics unravels molecular mechanisms in an ovine model. Dis Model Mech. 2018;11: dmm033092.

13. Shapiro F, Koide S, Glimcher MJ. Cell origin and differentiation in the repair of full-thickness defects of articular cartilage. J Bone Joint Surg Am. 1993;75:532-53.

14. Yu L, Dawson LA, Yan M, Zimmel K, Lin Y-L, Dolan CP, et al. BMP9 stimulates joint regeneration at digit amputation wounds in mice. Nat Commun. 2019;10:424.

15. Lee J, Marrero L, Yu L, Dawson LA, Muneoka K, Han M. SDF-1a/ CXCR4 signaling mediates digit tip regeneration promoted by BMP-2. Dev Biol. 2013;382:98-109.
16. Ide $\mathrm{H}$. Bone pattern formation in mouse limbs after amputation at the forearm level. Dev Dyn. 2012;241:435-41.

17. Ide H. Formation of distal bone elements in amputated neonatal mouse forelimbs. Integr J Orthop Traumatol. 2019;2:1-5.

18. Simkin J, Sammarco MC, Dawson LA, Schanes PP, Yu L, Muneoka K. The mammalian blastema: regeneration at our fingertips. Regeneration. 2015;2:93-105.

19. Han M, Yang X, Lee J, Allan CH, Muneoka K. Development and regeneration of the neonatal digit tip in mice. Dev Biol. 2008;315: 125-35.

20. Fernando WA, Leininger E, Simkin J, Li N, Malcom CA, Sathyamoorthi S, et al. Wound healing and blastema formation in regenerating digit tips of adult mice. Dev Biol. 2011;350:301-10.

21. Takeo M, Chou WC, Sun Q, Lee W, Rabbani P, Loomis C, et al. Wnt activation in nail epithelium couples nail growth to digit regeneration. Nature. 2013;499:228-32.

22. Yu L, Han M, Yan M, Lee J, Muneoka K. BMP2 induces segmentspecific skeletal regeneration from digit and limb amputations by establishing a new endochondral ossification center. Dev Biol. 2012;372:263-73.

23. Masaki H, Ide H. Regeneration potency of mouse limbs. Develop Growth Differ. 2007;49:89-98.

24. Chamberlain CS, Jeffery JJ, Leiferman EM, Yildirim T, Sun X, Baer GS, et al. Level-specific amputations and resulting regenerative outcomes in the mouse distal phalanx. Wound Repair Regen. 2017;25:443-53.

25. Miura S, Takahashi Y, Satoh A, Endo T. Skeletal callus formation is a nerve-independent regenerative response to limb amputation in mice and Xenopus. Regeneration. 2015;2:202-16.

26. Yu L, Han M, Yan M, Lee EC, Lee J, Muneoka K. BMP signaling induces digit regeneration in neonatal mice. Development. 2010;137:551-9.

27. Gardiner DM, Endo T, Bryant SV. The molecular basis of amphibian limb regeneration: integrating the old with the new. Semin Cell Dev Biol. 2002;13:345-52.

28. Suzuki M, Yakushiji N, Nakada Y, Satoh A, Ide H, Tamura K. Limb regeneration in Xenopus laevis froglet. Sci World J. 2006;6(Suppl 1):26-37.

29. Yokoyama H. Initiation of limb regeneration: the critical steps for regenerative capacity. Develop Growth Differ. 2008;50:13-22.

30. Goode RP. The regeneration of limbs in adult anurans. J Embryol Exp Morpholog. 1967;18:259-67.

31. Endo T, Tamura K, Ide H. Analysis of gene expressions during Xenopus forelimb regeneration. Dev Biol. 2000;220:296-306.

32. Tsutsumi R, Yamada S, Agata K. Functional joint regeneration is achieved using reintegration mechanism in Xenopus laevis. Regeneration. 2016;3:1-13.

33. Pacifici M, Koyama E, Iwamoto M. Mechanisms of synovial joint and articular cartilage formation: recent advances, but many lingering mysteries. Birth Defects Res C Embryo Today. 2005;75:23748.

34. Hunziker EB, Kapfinger E, Geiss J. The structural architecture of adult mammalian articular cartilage evolves by a synchronized process of tissue resorption and neoformation during postnatal development. Osteoarthr Cartil. 2007;15:403-13.

35. Decker RS, Um HB, Dyment NA, Cottingham N, Usami Y, Enomoto-Iwamoto $\mathrm{M}$, et al. Cell origin, volume and arrangement are drivers of articular cartilage formation, morphogenesis and response to injury in mouse limbs. Dev Biol. 2017;426:56-68.

36. Rux D, Decker RS, Koyama E, Pacifici M. Joints in the appendicular skeleton: developmental mechanisms and evolutionary influences. Curr Top Dev Biol. 2019;133:119-51.

37. Humason GL. Animal tissue techniques. San Francisco: Freeman; 1962. 
38. Lehoczky JA, Robert B, Tabin CJ. Mouse digit tip regeneration is mediated by fate-restricted progenitor cells. Proc Natl Acad Sci. 2011;108:20609-14.

39. Rinkevich Y, Lindau P, Ueno H, Longaker MT, Weissman IL. Germ-layer and lineage-restricted stem/progenitors regenerate the mouse digit tip. Nature. 2011;476:409-13.

40. Dawson LA, Yu L, Yan M, Marrero L, Schanes PP, Dolan C, et al. The periosteal requirement and temporal dynamics of BMP2induced middle phalanx regeneration in the adult mouse. Regeneration. 2017;4:140-50.

41. Dawson LA, Simkin J, Sauque M, Pela M, Palkowski T, Muneoka $\mathrm{K}$. Analogous cellular contribution and healing mechanisms following digit amputation and phalangeal fracture in mice. Regeneration. 2016;3:39-51.

42. Egawa S, Miura S, Yokoyama H, Endo T, Tamura K. Growth and differentiation of a long bone in limb development, repair and regeneration. Develop Growth Differ. 2014;56:410-24.

43. Van der Kraan PM, Buma P, Van Kuppevelt T, Van Den Berg WB. Interaction of chondrocytes, extracellular matrix and growth factors: relevance for articular cartilage tissue engineering. Osteoarthr Cartil. 2002;10:631-7.
44. Lyons KM, Rosen V. BMPs, TGF $\beta$, and border security at the interzone. Curr Top Dev Biol. 2019;133:153-70.

45. Rountree RB, Schoor M, Chen H, Marks ME, Harley V, Mishina $\mathrm{Y}$, et al. BMP receptor signaling is required for postnatal maintenance of articular cartilage. PLoS Biol. 2004;2:e355.

46. Gao L, Sheu T, Dong Y, Hoak DM, Zuscik MJ, Schwarz EM, et al. TAK1 regulates SOX9 expression in chondrocytes and is essential for postnatal development of the growth plate and articular cartilages. J Cell Sci. 2013;126:5704-13.

47. Pitsillides AA. Early effects of embryonic movement: "A shot out of the dark.". J Anat. 2006;208:417-31.

48. Archer CW, Dowthwaite GP, Francis-West P. Development of synovial joints. Birth Defects Res C Embryo Today. 2003;69:144-55.

49. Decker RS, Koyama E, Pacifici M. Genesis and morphogenesis of limb synovial joints and articular cartilage. Matrix Biol. 2014;39:510 .

Publisher's Note Springer Nature remains neutral with regard to jurisdictional claims in published maps and institutional affiliations. 